\title{
Rare lymphatic malformation
}

INSERM

\section{Source}

INSERM. (1999). Orphanet: an online rare disease and orphan drug data base. Rare

Iymphatic malformation. ORPHA:2415

Lymphatic malformation (LM), formerly referred to as lymphangioma, is a malformation localised to the lymphatic system. 